\title{
ESPAÇAMENTO PARA O CULTIVO DA BANANEIRA 'COMPRIDA VERDADEIRA' (Musa AAB) NA ZONA DA MATA SUL DE PERNAMBUCO $\left(1^{\circ} \text { CICLO }\right)^{1}$
}

\author{
ROBERTO JOSÉ MELLO DE MOURA², JOSUÉ FRANCISCO DA SILVA JUNIOR ${ }^{3 *}$, VENÉZIO FELIPE DOS SANTOS ${ }^{2}$, \\ JANDELSON GOUVEIA ${ }^{4}$
}

\begin{abstract}
RESUMO - O presente trabalho objetivou definir o melhor espaçamento no desenvolvimento e rendimento da bananeira- 'Comprida Verdadeira' na Zona da Mata Sul de Pernambuco. O experimento foi conduzido em delineamento de blocos ao acaso, com três tratamentos, constituídos por três diferentes espaçamentos $(3,0$ × 2,0 m, 2,5 x 2,0 m e 2,0 x 2,0 m) e três repetições. Foram avaliados a altura da planta, circunferência do pseudocaule, número de filhos e folhas emitidos, número de dias do plantio à emissão da inflorescência e colheita, peso do cacho e das pencas, número de frutos e de pencas/cacho, comprimento e diâmetro do fruto, e espessura da casca. As diferentes distâncias entre plantas não influenciaram na produtividade do primeiro ciclo. Entretanto, os espaçamentos 3,0 x 2,0 m e 2,5 x 2,0 m promoveram a produção de cachos maiores, bem como frutos com melhores características físicas.
\end{abstract}

Termos para Indexação: Banana, "plátano”, densidade de plantio, distância entre plantas, sistema de cultivo, manejo de touceiras.

\section{SPACING ON 'COMPRIDA VERDADEIRA' PLANTAIN (Musa AAB) CULTIVATATED IN THE SOUTH RAIN FOREST REGION OF PERNAMBUCO STATE, BRAZIL (1st. CYCLE)}

\begin{abstract}
This work aimed to define adequate spacings for the development and yield of 'Comprida Verdadeira' plantain in the South Rain Forest Region of Pernambuco State, Brazil. Experimental design of randomized bloks with three treatments, composed of three distinct spacings ( 3.0 x $2.0 \mathrm{~m}$, $2.5 \times 2.0 \mathrm{~m}$ and $2.0 \times 2.0 \mathrm{~m}$ ), and three replicates were utilized. The characteristics evaluated were: plant height, pseudostem circunference, numbers of suckers and leaves emitted, days from planting to inflorescence emission and harvest, bunch and hands weights, numbers of fruits and hands per bunch, fruit lenght and diameter, and skin thickness. Different distances had no effect on first cycle productivity. However, 3.0 x $2.0 \mathrm{~m}$ and $2.5 \mathrm{x} 2.0 \mathrm{~m}$ spacings increased yield of bunch weight, and fruits showed the best physical characteristics.
\end{abstract}

Index Terms: Banana, planting density, planting distance, cultivation system, sucker management.

\section{INTRODUÇÃO}

O Brasil destaca-se como o terceiro produtor mundial de banana, com uma produção de 6,34 milhões de toneladas, segundo dados da FAO (2001). Não se sabe, entretanto, quanto desse total corresponde às bananas de cozinhar e fritar do grupo $\mathrm{AAB}$, conhecidas mundialmente como "plátanos". É certo que as regiões Nordeste e Norte concentram os maiores plantios desse tipo de banana, que é importante fonte de alimento para todas as camadas sociais da população. Embora Pernambuco seja um dos maiores produtores de banana do País [36,9 milhões de cachos, em 2000, conforme IBGE (2001)], cerca de $86 \%$ das bananas do tipo "plátano" que consome, são importadas de outros Estados, como Alagoas e Bahia (Ceagepe, 1996). Existem poucas informações acerca do cultivo desse tipo de banana no Brasil, e o uso de tecnologias é extrapolado a partir de outras variedades. Com relação ao manejo de touceiras, os estudos são ainda exíguos, possivelmente devido aos problemas peculiares da própria conformação genética do subgrupo Plátano (afloramento de rizoma e elevada suscetibilidade à broca-do-rizoma e nematóides), que faz com que a condução das plantas se torne bastante trabalhosa.

A maioria dos trabalhos sobre densidade de plantio e espaçamento foi realizada em outros países, sobretudo da América Latina e África. Tézenas du Montcel (1987) recomenda, para os países africanos, densidades que vão de 1.500 até 3.000 plantas/ha, dependendo do tipo de "plátano" cultivado. O espaçamento pode variar de 2,0 x 1,8 m até $3,0 \times 2,0 \mathrm{~m}$, sendo usado o sistema tradicional de condução de touceiras. Añez et al. (1991) testaram na Venezuela 11 diferentes distâncias de plantio e concluíram que os rendimentos do "plátano" 'Hartón' aumentaram proporcionalmente com o aumento das distâncias. $\mathrm{Na} \mathrm{Co}$ lômbia, Belalcazar Carvajal et al. (1994a, 1994b) constataram que, para o "plátano" 'Dominico Hartón', o espaçamento de 3,0 x 2,0 m, e a densidade de 1.666 plantas/ha, com um rebento por touceira em explorações perenes, ou as altas densidades no mesmo espaçamento (3.332 e 4.490 plantas/ha com duas e três plantas por touceira, respectivamente), em explorações anuais, são as mais recomendadas para obtenção de elevadas produções. No Brasil, em ensaio para definição de espaçamento, adubação e calagem da planta-mãe da bananeira- 'Comprida Verdadeira', Cavalcante et al. (1981) encontraram melhores resultados na distância de 3,0 x 2,0 m, entre três espaçamentos testados (3,0 x 2,0 a 4,0 m). Alves \& Oliveira (1999) recomendam densidades de 1.111 a 3.333 plantas/ha para as cultivares Terra e Maranhão, e 1.666 a 4.998 plantas/ha para 'd'Angola' e 'Terrinha', com dois seguidores.

O objetivo deste trabalho foi definir o melhor espaçamento no desenvolvimento e rendimento da bananeira- 'Comprida Verdadeira' na Zona da Mata Sul de Pernambuco.

\section{MATERIALEMÉTODOS}

O experimento foi desenvolvido no Engenho Amorinhas, localizado no Município de Amaraji, na Zona da Mata Sul de Pernambuco, que se situa a $8^{\circ} 22^{\prime} 45^{\prime}$ 'S, $35^{\circ} 27^{\prime} 00^{\prime \prime} \mathrm{W}$ e a uma altitude de $180 \mathrm{~m}$. O clima da região é tropical quente e úmido, de transição entre As' e Ams' (classificação de Köppen), com pluviosidade e temperatura médias anuais de $2.007 \mathrm{~mm}$ e $24^{\circ} \mathrm{C}$, respectivamente. O solo é do tipo Latossolo Vermelho-Amarelo distrófico. O material genético utilizado foi a bananeira tipo French 'Comprida Verdadeira' (Musa AAB, subgrupo Plátano), também conhecido como 'Samburá' ou 'Sete Palmas', sendo a cultivar desse subgrupo mais plantada no Estado. O delineamento experimental foi o de blocos ao acaso, com três tratamentos, constituídos por três espaçamentos $(3,0$ x 2,0 m, 2,5 x 2,0 m e 2,0 x 2,0 m) e três repetições. Cada parcela foi formada por 35 plantas (espaçamentos 2,5 x 2,0 m e 2,0 x 2,0 m) e 28 plantas (espaçamento 3,0 x 2,0 m), sendo 15 consideradas úteis no espaçamento $2,0 \times 2,0 \mathrm{~m}, 10$ no espaçamento $3,0 \times 2,0 \mathrm{~m}$ e 12 no espaçamento $2,5 \times 2,0 \mathrm{~m}$, de modo que a área útil de cada parcela foi de 60 $\mathrm{m}^{2}$, independentemente do tratamento.

O plantio foi realizado em covas de $0,40 \times 0,40 \times 0,40 \mathrm{~m}$ e as

1 (Trabalho 036/2002). Recebido: 09/03/2002. Aceito para publicação: 26/09/2002.

Pesquisa financiada pelo Programa Nacional de Fortalecimento da Agricultura Familiar - PRONAF;

2 Eng. Agrônomo, B.Sc., Empresa Pernambucana de Pesquisa Agropecuária - IPA, Caixa Postal 1022, CEP 50761-000 - Recife, PE. E-mail: roberto@ipa.br;

3 Eng. Agrônomo, M.Sc., Embrapa Tabuleiros Costeiros, Caixa Postal 44, CEP 49025-040- Aracaju, SE. E-mail: josue @ cpatc.embrapa.br;

4 Eng. Agrônomo, B.Sc., Secretaria de Agricultura de Primavera, Rua Cap. Lima Ribeiro, CEP 55510-000 - Primavera, PE. E-mail: jandelsongouveia@ uol.com.br.

* Autor para correspondência 
mudas utilizadas foram do tipo pedaço de rizoma, com aproximadamente $1,0 \mathrm{~kg}$. As plantas foram conduzidas sob regime de irrigação por aspersão sobcopa e submetidas aos tratos culturais e fitossanitários recomendados para a cultura (Alves \& Oliveira, 1999). As fertilizações mineral e orgânica foram realizadas conforme recomendação de Gonzaga Neto et al. (1998) para uso em bananeira irrigada no Estado de Pernambuco. Foram avaliadas as seguintes características: altura da planta no florescimento; número de filhos e de folhas até a colheita; número de dias do plantio ao florescimento e à colheita; peso do cacho e das pencas, número de pencas e frutos por cacho; comprimento e diâmetro do fruto e espessura da casca. Os dados foram submetidos à análise estatística e as médias foram comparadas pelo teste de Tukey, ao nível de 5\% de probabilidade.

\section{RESULTADOSE DISCUSSÃO}

Com respeito ao número de dias do plantio à emissão da inflorescência e até a colheita, não houve diferença entre os tratamentos estudados (Tabela 1). Em relação à altura da planta, aquelas conduzidas no espaçamento 3,0 × 2,0 m alcançaram o maior porte $(2,70 \mathrm{~m})$, sem diferir daquelas cultivadas no espaçamento 2,5 x 2,0 m (2,67 m). Já no tratamento mais adensado, o porte foi mais reduzido $(2,54 \mathrm{~m})$. Comportamento semelhante foi observado para a circunferência do pseudocaule. As bananeiras conduzidas em espaçamentos maiores podem ter sofrido maior incidência de luz, o que promoveu mais desenvolvimento das plantas, sem que o crescimento estivesse associado a estiolamento, como pode ser comprovado pelos valores da circunferência do pseudocaule. Essa mesma explicação pode ser aplicada a algumas características de produção e físicas do fruto mencionadas adiante.

TABELA 1 - Características de desenvolvimento da bananeira- 'Comprida Verdadeira' cultivada sob diferentes espaçamentos $\left(1^{\circ}\right.$ ciclo), Amaraji-PE, 2000.

\begin{tabular}{ccccccc}
\hline Espaçamento & $\begin{array}{c}\mathbf{N}^{\circ} \text { de dias até } \\
\text { emissão da } \\
\text { inflorescência }\end{array}$ & $\begin{array}{c}\mathbf{N}^{\circ} \text { de dias } \\
\text { do plantio } \\
\text { à colheita }\end{array}$ & $\begin{array}{c}\text { Altura da } \\
\text { planta } \\
(\mathbf{m})\end{array}$ & $\begin{array}{c}\text { Circunferência } \\
\text { do pseudocaule } \\
(\mathbf{c m})\end{array}$ & $\begin{array}{c}\mathbf{N}^{\circ} \text { de } \\
\text { filhos até }\end{array}$ & $\begin{array}{c}\mathbf{N}^{\circ} \text { de } \\
\text { a colheita }\end{array}$ \\
\hline $2,0 \times 2,0 \mathrm{~m}$ & $333,4 \mathrm{a}$ & $431,8 \mathrm{a}$ & $2,70 \mathrm{a}$ & 65,26 & $6,8 \mathrm{a}$ & $26,8 \mathrm{a}$ \\
$2,5 \times 2,0 \mathrm{~m}$ & $321,6 \mathrm{a}$ & $429,3 \mathrm{a}$ & $2,67 \mathrm{ab}$ & $62,84 \mathrm{ab}$ & $6,8 \mathrm{a}$ & $26,2 \mathrm{a}$ \\
$3,0 \times 2,0 \mathrm{~m}$ & $324,1 \mathrm{a}$ & $429,1 \mathrm{a}$ & $2,54 \mathrm{~b}$ & $60,28 \mathrm{~b}$ & $7,0 \mathrm{a}$ & $26,5 \mathrm{a}$ \\
\hline
\end{tabular}

Médias seguidas pela mesma letra não diferem significativamente entre si, pelo teste de Tukey, a 5\% de probabilidade

Não houve diferença para número de filhos emitidos até a colheita e de folhas em nenhum espaçamento. No que concerne às características de rendimento (Tabela 2), não houve diferença na produtividade, mesmo quando foi utilizado o sistema de condução mais adensado; entretanto, quando se avaliou a produção em peso de cacho por planta, observou-se que as plantas no espaçamento 3,0 x 2,0 m alcançaram o valor mais alto $(17,8 \mathrm{~kg})$, diferindo significativamente das plantas no espaçamento 2,0 x 2,0 m, que obtiveram uma produção de 14,4 kg. Esse mesmo comportamento foi observado para o peso das pencas. Em relação ao número de pencas e de frutos por cacho, não foi encontrada diferença entre os tratamentos. Resultados semelhantes também foram obtidos por Reyes \& Armijos (1998) para o "plátano" 'Barraganete', no Equador, onde os cachos de menor peso também foram obtidos em plantios mais adensados. Em avaliações realizadas na Colômbia por Belalcázar Carvajal \& Cayón Salinas (1998), verificou-se que, no primeiro ciclo de produção, o "plátano" 'Dominico Hartón' cultivado no espaçamento 3,0 x $2,0 \mathrm{~m}$, com uma planta por touceira, alcançou uma produtividade de $23,2 \mathrm{t} / \mathrm{ha}$, enquanto sob altas densidades registraram-se valores de até $51,8 \mathrm{t} / \mathrm{ha}$, Os cachos obtidos em plantios muito adensados apresentaram menores pesos em todos os ciclos de produção.

Quanto às características físicas do fruto (Tabela 3), notou-se que, para o peso do fruto, as plantas conduzidas na distância 3,0 x 2,0 m obtiveram a maior média $(208,50 \mathrm{~g})$, que não diferiu das plantas no espaçamento 2,5 x 2,0 m (193,48 g), mas que foi sensivelmente maior que as do 2,0 x 2,0 m (170,68 g), havendo diferença entre os mesmos. Comportamento similar foi registrado para comprimento e diâmetro do fruto, para os quais as plantas do espaçamento 3,0 x 2,0 m alcançaram os maiores valores médios ( $23,34 \mathrm{~cm}$ e $39,15 \mathrm{~mm}$, respectivamente), embora não tenham diferido das plantas na distância 2,5 x 2,0 m, que obtiveram para essas mesmas variáveis os valores de $22,70 \mathrm{~cm} \mathrm{e} 36,60 \mathrm{~mm}$, respectivamente. Os frutos avaliados nas plantas do espaçamento $2,0 \times 2,0 \mathrm{~m}$ apresentaram os menores comprimento e diâmetro $(21,34 \mathrm{~cm}$ e $36,67 \mathrm{~mm}$, respectivamente), diferindo significativamente dos demais. A espessura da casca não apresentou diferença entre todos os tratamentos.

TABELA 2 - Características de produção da bananeira- 'Comprida Verdadeira' cultivada sob diferentes espaçamentos $\left(1^{\circ}\right.$ ciclo $)$, Amaraji-PE, 2000.

\begin{tabular}{lccccc}
\hline Espaçamento & $\begin{array}{c}\text { Peso do } \\
\text { cacho (kg) }\end{array}$ & $\begin{array}{c}\text { Peso das } \\
\text { pencas (kg) }\end{array}$ & $\begin{array}{c}\text { Produtividade } \\
\text { (t/ha) }\end{array}$ & $\begin{array}{c}\mathbf{N}^{\circ} \text { de } \\
\text { pencas/ } \\
\text { cacho }\end{array}$ & $\begin{array}{c}\mathbf{N}^{\circ} \text { de frutos/ } \\
\text { cacho }\end{array}$ \\
\hline $2,0 \times 2,0 \mathrm{~m}$ & $14,4 \mathrm{~b}$ & $13,2 \mathrm{~b}$ & $34,1 \mathrm{a}$ & $6,7 \mathrm{a}$ & $78,0 \mathrm{a}$ \\
$2,5 \times 2,0 \mathrm{~m}$ & $17,1 \mathrm{ab}$ & $15,8 \mathrm{ab}$ & $34,0 \mathrm{a}$ & $6,9 \mathrm{a}$ & 81,2 \\
$3,0 \times 2,0 \mathrm{~m}$ & $17,8 \mathrm{a}$ & $16,7 \mathrm{a}$ & $30,2 \mathrm{a}$ & $7,0 \mathrm{a}$ & 83,9 \\
\hline
\end{tabular}

Médias seguidas pela mesma letra não diferem significativamente entre si, pelo teste de Tukey, a $5 \%$ de probabilidade

TABELA 3 - Características físicas do fruto da bananeira- 'Comprida Verdadeira' cultivada sob diferentes espaçamentos $\left(1^{\circ}\right.$ ciclo), Amaraji-PE, 2000.

\begin{tabular}{ccccc}
\hline Espaçamento & $\begin{array}{c}\text { Peso do fruto } \\
(\mathrm{g})\end{array}$ & $\begin{array}{c}\text { Comprimento } \\
\text { do fruto }(\mathbf{c m})\end{array}$ & $\begin{array}{c}\text { Diâmetro do } \\
\text { fruto }(\mathbf{m m})\end{array}$ & $\begin{array}{c}\text { Espessura da } \\
\text { casca }(\mathbf{m m})\end{array}$ \\
\hline $2,0 \times 2,0 \mathrm{~m}$ & $170,68 \mathrm{~b}$ & $21,34 \mathrm{~b}$ & $36,67 \mathrm{~b}$ & $2,42 \mathrm{a}$ \\
$2,5 \times 2,0 \mathrm{~m}$ & $193,48 \mathrm{ab}$ & $22,70 \mathrm{a}$ & $38,60 \mathrm{a}$ & 2,55 \\
$3,0 \times 2,0 \mathrm{~m}$ & $208,50 \mathrm{a}$ & $23,34 \mathrm{a}$ & $39,15 \mathrm{a}$ & 2,67 \\
\hline
\end{tabular}

Médias seguidas pela mesma letra não diferem significativamente entre si, pelo teste de Tukey, a 5\% de probabilidade

\section{CONCLUSÃO}

Nas condições do presente estudo, os espaçamentos 3,0 × 2,0 $\mathrm{m}$ e 2,5 x 2,0 m promovem a produção de cachos maiores, bem como de frutos com melhores características físicas (maiores peso e tamanho) no primeiro ciclo de cultivo da bananeira- 'Comprida Verdadeira', sem prejudicar a produtividade, quando comparados ao espaçamento 2,0 × 2,0m.

\section{REFERÊNCIASBIBLIOGRÁFICAS}

ALVES, E.J.; OLIVEIRA, M. A. de. Planejamento de um plantio comercial. In: ALVES, E.J. (Coord.) A cultura da banana: aspectos técnicos, socioeconômicos e agroindustriais. 2.ed. Brasília, DF: EMBRAPASPI/Cruz das Almas, BA: EMBRAPA-CNPMF, 1999. p. 261-290.

AÑEZ, B.; TAVIRA, E.; SALAS, J.A. Efecto de la distancia entre hileras sobre la producción de plátano. In: REUNIÓN DE LA ACORBAT, 9., 1989, Mérida, Venezuela. Memorias... Maracaibo: ACORBAT, 1991. p. 457-471.

BELALCAZAR CARVAJAL, S.; VALENCIA M., J.A.; ARCILA P., M.I. Estudio sobre las densidades de población em plátano clon Dominico-Hartón (Musa AAB, Simmonds) en Colombia. In: REUNIÓN DE LA ACORBAT, 10., 1991, Villahermosa, Mexico. Memorias... San José de Costa Rica: CORBANA, 1994a. p. 535-548.

BELALCAZAR CARVAJAL, S.; CAYON SALINAS, D.G. Altas densidades de siembra. In: SEMINÁRIO INTERNACIONAL SOBRE PRODUCCIÓN DE PLÁTANO, 1998, Arménia, Colômbia. Memórias... Arménia, Colômbia: Corpoica/ Universidad del Quíndio/ SENA/ Comité Departamental de Cafeteros del Quíndio. Disponível em: 
<http://www.uniquindio.edu.co/infoadmi/platano/memorias.htm.> Acesso: em 29 de agosto de 2002

CAYON, D.G.; FRANCO, G. Altas densidades de siembra. InfoMusa, Montpellier, v.3, n.1, p.12-25, jun. 1994b.

CAVALCANTE, A.T.; MOURA, R.J.M. de; MAAZE, U.C.; REIS, O.V. dos. Efeito de espaçamento, adubação e calagem em bananeira-Comprida (planta-mãe). Atuação do IPA no âmbito do Pólo Nordeste. Recife, PE: IPA, 1981. v.1, n.p.

GONZAGA NETO, L.; PEREIRA, J.R.; SILVA, D.J. Banana irrigada (Musa spp.). CAVALCANTI, F.J. de A. (Coord.) Recomendações de adubação para o Estado de Pernambuco: $2^{a}$ aproximação. 2. ed. Recife, PE: IPA, 1998. p. 115.

CEAGEPE (Companhia de Abastecimento e de Armazéns Gerais do Estado de Pernambuco). Análise conjuntural do mercado a nível de atacado na unidade CEASA-PE: período 1986 a 1995. Recife: Bagaço, 1996. p. 125-144.

FAO. Statistical databases (agriculture) - 2000. Roma, 2001. Disponível em: <http://www.fao.org.> Acesso em: 20 de outubro de 2001.

IBGE. Levantamento sistemático da produção agrícola - 2000. Rio de Janeiro, 2001. Disponível em: <http://www.sidra.ibge.gov.br.> Acesso em: 20 de outubro de 2001.

REYES, W.; ARMIJOS L., F. Densidades de siembra en plátano cv. Barraganete (AAB)enElCarmen, Ecuador. In: REUNIÓNDELA ACORBAT, 13., 1998, Guayaquil. Memorias... Guayaquil: CONABAN, 1998. p. 68-69. TÉZENAS DU MONTCEL, H. Plantain bananas. London: CTA/McMillan, 1987. 106p. (Série The Tropical Agriculturist). (Original em francês. Tradução de Paul Skinner). 